\title{
DARMKREBS
}

\section{Bessere Früherkennung mit ASS?}

Die Einnahme niedrig dosierter Acetylsalicylsäure (ASS) verschlechtert die Zuverlässigkeit von Tests auf okkultes Blut im Stuhl nicht - im Gegenteil: Einer Studie von $\mathrm{H}$. Brenner et al. vom Deutschen Krebsforschungszentrum zufolge erhöht sie sogar die Sensitivität des Tests. In der Studie wurden 1979 Patienten, die regelmäßig niedrig dosierte ASS einnahmen, und 1746 Personen ohne ASS-Prophylaxe im Rahmen des Krebsfrüherkennungsprogramms mittels Stuhltest untersucht. ASS verbes- serte die Treffsicherheit der Tests erheblich: Fortgeschrittene Adenome wurden im Hämoglobin-Test in der ASS-Gruppe mit 70,8\% gegenüber $35,9 \%$ in der NichtASS-Gruppe erkannt. Offensichtlich werden Krebsvorstufen durch ASS zum Bluten angeregt, wodurch sich die Detektionschance erhöht. Die Angst vor falsch-positiven Tests bei Einnahme von ASS scheint dagegen unbegründet: Die Spezifität sank nur unwesentlich von $91,1 \%$ auf $85,7 \%$. JAMA 2010;304(22):2513-2520

\section{IM KINDERGARTEN DAUERND KRANK ... \\ In der Grundschule dann um so gesünder}

Kinder, die Kinderkrippen bzw. Kindergärten besuchen, haben häufig Atemwegsund Ohrinfektionen. Befürchtungen, dass diese Kinder auch später häufiger krank sind, hat jetzt eine kanadische Studie zerstreut.

Forscher der Universität Montreal ließen sich von 1238 Familien, in denen 1998 ein Kind zur Welt gekommen war, acht Jahre lang über alle Atemwegs-, Ohr- und gastrointestinalen Infektionen informieren.
Kinder, die in Einrichtungen mit vielen anderen Kindern untergebracht waren, hatten im Alter von 2,5 Jahren häufiger Atemwegs- und Ohrinfektionen. Dafür waren sie dann im Alter von sechs bis acht Jahren gesünder als andere Kinder. Bei den gastrointestinalen Infektionen zeigte sich kein Zusammenhang zwischen Kindergartenbesuch und Erkrankungshäufigkeit.

Archives of Pediatrics \& Adolescent Medicine, 2010; DOI: 10.1001/archpediatrics.2010.216

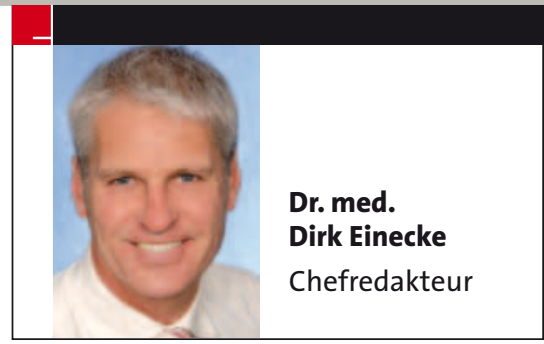

NACH DEM HERZINFARKT

\section{Fragen Sie nach Suizidgedanken}

Die Autoren einer dänischen Studie empfehlen, Herzinfarktpatienten routinemäßig auf das Vorhandsein von Suizidgedanken zu screenen. Sie berufen sich dabei auf die Auswertung von fünf Registern, aus denen sie 19857 Selbstmordfälle herausfilterten. Demnach hatten Herzinfarktpatienten ohne psychiatrische Vorgeschichte ein dreimal höheres Risiko, einen Suizid zu begehen, als eine Vergleichsgruppe ohne Infarkt. Berücksichtigte man auch $\mathrm{Pa}$ tienten mit bekannter psychischer Störung vor dem Infarkt, erhöhte sich das Suizidrisiko sogar um den Faktor 64. Das Selbstmordrisiko war in den ersten vier Wochen nach dem Myokardinfarkt am größten. Aber auch nach fünf Jahren war die Suizidrate noch erhöht.

Larsen KK, et al. Circulation 2010;122:2388-2393

\section{DA STECKT MEHR DAHINTER}

\section{Neuer Internet-Auftritt für die MMW}

Ab sofort finden Sie Ihre MMW online auf dem Internetportal SpringerMedizin. Sie können sie wie gewohnt unter der Internetadresse mmw.de aufrufen. Wie bisher steht Ihnen neben der aktuellen Ausgabe der MMW auch das MMW-Archiv zur Verfügung. Nach der Registrierung auf SpringerMedizin.de, dem Fachportal für Ärzte, erwarten Sie aber noch viele weitere nützliche Informationen für Ihren beruflichen Alltag aus der ganzen Welt von SpringerMedizin. Melden Sie sich noch heute kostenfrei an!

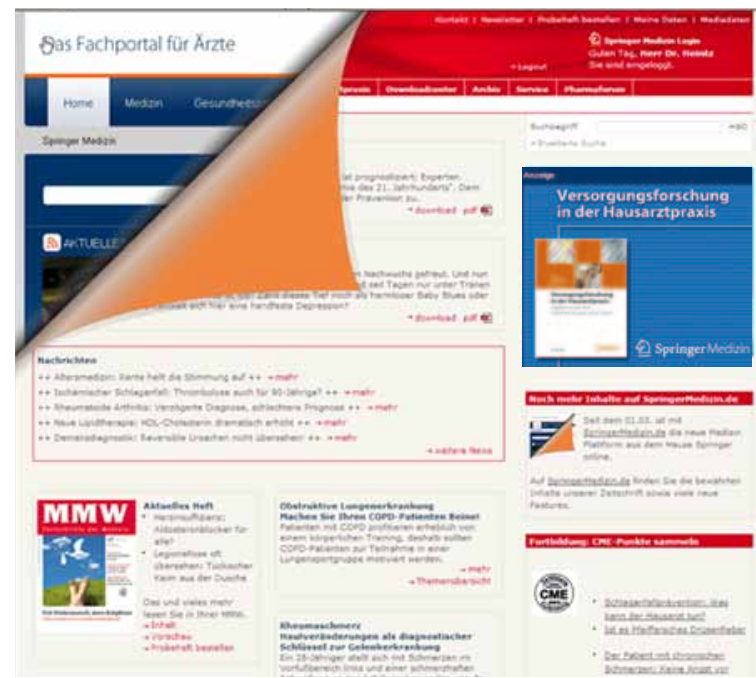

